\title{
BMJ Open Pregnancy outcomes of early detected gestational diabetes: a retrospective comparison cohort study, Qatar
}

\author{
Mohammed Bashir, ${ }^{1}$ Khaled Baagar, ${ }^{1}$ Emad Naem, ${ }^{1}$ Fadi Elkhatib, ${ }^{2}$ \\ Noor Alshaybani, ${ }^{2}$ Justin C Konje, ${ }^{3}$ Abdul-Badi Abou-Samra ${ }^{1}$
}

To cite: Bashir M, Baagar K, Naem E, et al. Pregnancy outcomes of early detected gestational diabetes: a retrospective comparison cohort study, Qatar. BMJ Open 2019;9:e023612. doi:10.1136/ bmjopen-2018-023612

- Prepublication history for this paper is available online. To view these files, please visit the journal online (http://dx.doi. org/10.1136/bmjopen-2018023612).

Received 16 April 2018 Revised 15 0ctober 2018 Accepted 21 December 2018

\section{Check for updates}

C Author(s) (or their employer(s)) 2019. Re-use permitted under CC BY-NC. No commercial re-use. See rights and permissions. Published by BMJ.

${ }^{1}$ Department of Endocrinology, Qatar Metabolic Institute, Hamad Medical Corporation, Doha,

Qatar

${ }^{2}$ Department of Obstetrics and Gynaecology, Women's Hospital, Hamad Medical Corporation, Doha, Qatar

${ }^{3}$ Department of Obstetrics and Gynaecology, Sidra Medical, Doha, Qatar

Correspondence to Mohammed Bashir; mohdbashir80@hotmail.com

\section{ABSTRACT}

Objective To compare pregnancy outcomes in patients with early versus usual gestational diabetes mellitus (GDM).

Design A retrospective cohort study.

Settings The Women's Hospital, Hamad Medical Corporation, Qatar.

Participants GDM women who attended and delivered in the Women's Hospital, between January and December 2016. GDM was diagnosed based on the 2013-WHO criteria. The study included 801 patients; of which, 273 E-GDM and 528 U-GDM. Early GDM (E-GDM) and usual GDM (U-GDM) were defined as GDM detected before and after 24 weeks' gestation, respectively.

Outcomes Maternal and neonatal outcomes and the impact of timing of GDM-diagnosis on pregnancy outcomes.

Results At conception, E-GDM women were older (mean age $33.5 \pm 5.4$ vs $32.0 \pm 5.4$ years, $p<0.001)$ and had higher body mass index $\left(33.0 \pm 6.3\right.$ vs $\left.31.7 \pm 6.1 \mathrm{~kg} / \mathrm{m}^{2}, \mathrm{p}=0.0059\right)$ compared with U-GDM. The mean fasting, and 1-hour blood glucose levels were significantly higher in E-GDM vs U-GDM, respectively $(5.3 \pm 0.7$ vs $4.0 \pm 0.7 \mathrm{mmol} / \mathrm{L}$, $p<0.001$ and $10.6 \pm 1.7$ vs $10.3 \pm 1.6 \mathrm{mmol} / \mathrm{L}, p<0.001$ ). More patients in the U-GDM were managed on diet alone compared with E-GDM ( $53.6 \%$ vs $27.5 \%, \mathrm{p}<0.001)$. E-GDM subjects gained less weight per week compared with U-GDM $(0.02 \pm 0.03$ vs $0.12 \pm 0.03 \mathrm{~kg} /$ week, $p=0.0274)$. Maternal outcomes were similar between the two groups apart from a higher incidence of preterm labour $(25.5 \%$ vs $14.4 \% ; p<0.001)$ and caesarean section (52.4\% vs $42.8 \%$; $p=0.01$ ) in $E-G D M$ vs $U-G D M$, respectively. After correction for covariates; gestational age at which GDM was diagnosed was associated with increased risk of macrosomia (OR 1.06, $95 \% \mathrm{Cl} 1.00$ to $1.11 ; p<0.05)$ and neonatal hypoglycaemia (OR 1.05, $95 \% \mathrm{Cl} 1.00$ to $1.11 ; p<0.05)$.

Conclusion Our data support the concept of early screening and treatment of GDM in high-risk patients. More data are needed to examine the optimal time for screening.

\section{INTRODUCTION}

Gestational diabetes mellitus (GDM) is defined as hyperglycaemia first detected during pregnancy that is clearly not type 1 diabetes mellitus (T1DM) or type 2 diabetes

\section{Strength and limitations of this study}

- The main limitation of our study is the retrospective design.

- The duration between diagnosis and intervention was not available.

- The rate of gestational weight gain in each trimester was not available.

- The major strength is the large number of the study population and the low level of missing data.

- It is a single-centre study, all patients were treated with the same medical team using standardised protocols.

mellitus (T2DM). ${ }^{1}$ There are not too many areas of diabetes that have generated as much debate, controversy and lack of consensus as GDM. The debates cover the diagnostic criteria, classification, timing of screening and method of screening (universal vs selective screening). ${ }^{1-4}$ The HAPO trial (hyperglycaemia and adverse pregnancy outcomes) examined 25505 pregnancies who underwent $75 \mathrm{~g}$ OGTT (oral glucose tolerance test) between 24 and 32 weeks' gestation and showed an association between fasting, 1 hour and 2 hours blood glucose and the risk of large for gestational age (LGA). ${ }^{5}$ Most of the current glucose threshold for the diagnosis of GDM are driven from the HAPO trial and are only validated between 24 and 32 weeks' gestation. Most of the current guidelines, however, support early screening for hyperglycaemia in pregnancy to detect cases of undiagnosed T2DM. Because of this early screening, there is a group of patients who do not have overt diabetes but fulfil the criteria for the diagnosis of GDM; a group which is currently known as early detected GDM (E-GDM) as opposed to usual GDM (U-GDM) which is detected after 24 weeks' gestation.

There are currently neither an agreement on the existence of E-GDM nor on the glucose threshold for its diagnosis. For example, the 
American Diabetes Association (ADA) does not acknowledge the existence of E-GDM, while the WHO advocates that GDM could be diagnosed at any time during pregnancy using the same glucose threshold. ${ }^{16}$ Conflicting data emerged from few studies that compared pregnancy outcomes of E-GDM with U-GDM. Sweeting et al reported that patients with GDM diagnosed prior to 12 weeks' gestation had pregnancy outcomes similar to pre-existing DM, while patients with GDM diagnosed between 12 and 23 weeks of gestation had more frequent hypertensive disorders compared with those diagnosed between 24 and 28 weeks' gestation. ${ }^{7}$ Another study compared 284 patients with GDM diagnosed before and after 20 weeks' gestation and found no difference in pregnancy outcomes. ${ }^{8}$ On the other hand, a recent meta-analysis of cohort studies showed that many patients with early diagnosed GDM had normal glucose values when they were rescreened between 24 and 28 weeks' gestation questioning the screening process for E-GDM. ${ }^{9}$

Qatar is a growing urban country in the Middle-East with a high prevalence of obesity and T2DM. ${ }^{10}$ Qatar has implemented a national programme to screen all pregnant ladies for diabetes at the first antenatal care visit using fasting blood glucose (FBG). Early screening for diabetes in pregnancy-which is primarily performed to rule out pre-existing diabetes-allowed the diagnosis of E-GDM. This study reports the outcomes of E-GDM versus U-GDM in Qatar.

\section{METHODS}

This was a retrospective cohort study which was undertaken at the Women's Hospital of Hamad Medical Corporation (HMC), Doha, Qatar. All pregnant women are screened in the first antenatal care visit using FBG. If the FBG at the first visit is normal, $75 \mathrm{~g}$ OGTT is performed at 24 weeks' gestation. In high-risk patients, the $75 \mathrm{~g}$ OGTT is performed at 16 weeks and, if normal, repeated at 24 weeks' gestation. High-risk patients are defined as women with a history of GDM; women with a history of impaired fasting glucose and/or impaired glucose tolerance; women with a history of unexplained stillbirth, Intrauterine fetal death (IUFD) or unexplained neonatal death or birth of baby with malformations associated with diabetes; women with history of macrosomic baby weighing $>4 \mathrm{~kg}$; women with a history of polycysitc ovary syndrome (PCOS) and women with body mass index $(\mathrm{BMI})>25 \mathrm{~kg} / \mathrm{m}^{2}$. The WHO criteria $(\mathrm{FBG} \geq 5.1 \mathrm{mmol} / \mathrm{L}$, 1 hour post OGTT $\geq 10.0 \mathrm{mmol} / \mathrm{L}$ or 2 hours post OGTT $\geq 8.5 \mathrm{mmol} / \mathrm{L}$ ) is used to diagnose GDM at any time of pregnancy. All GDM patients are managed by dietary modification and if $20 \%$ or more of the self-monitoring blood glucose readings are above the ADA targets (FBG $\leq 5.3 \mathrm{mmol} / \mathrm{L}$, I-hour post prandial $\leq 7.8 \mathrm{mmol} / \mathrm{L}$ or 2 hours $\leq 6.7 \mathrm{mmol} / \mathrm{L}$ ), the women are referred to the Diabetes in Pregnancy Clinic at the Women's hospital. The Women's Hospital is the largest maternity hospital in the country overseeing between 16 and 18 thousand deliveries per year. Metformin is the first-line medical therapy for all patients with GDM who do not respond to dietary control unless it is contraindicated, unacceptable to the patient or is not tolerated. Insulin is used as supplementary to metformin or solely if metformin was not tolerated or could not be used.

We report on 801 patients with GDM who were managed and delivered in the Women's hospital between January and December 2016. We defined E-GDM and U-GDM as GDM that was diagnosed before and after 24 weeks' gestation, respectively. Prepregnancy weight is recorded in the first visit based on patient self-report and is identified on the electronic medical records as "prepregnancy weight'. If the prepregnancy weight was not recorded, we used the last recorded weight before conception otherwise the weight is considered as 'missing'. We recorded the last height recorded before conception or the height recorded in the first antenatal visit. Maternal age was calculated as the age of the mother at conception. Macrosomia is defined as birth weight $>4000 \mathrm{~g}$; low birth weight was defined as birth weight $<2500 \mathrm{~g}$; LGA was defined as birth weight $>90$ th percentile; small for gestational age (SGA) is defined as birth weight $<10$ th percentile; and preterm delivery was defined as delivery before the 37 th week of gestation. We calculated the average weekly gestational weight gain (wGWG)

$$
\frac{\text { weight at delivery }(\mathrm{kg}) \text {-weight at conception }(\mathrm{kg})}{\text { Gestational age at delivery }(\text { weeks })}
$$

\section{Statistics}

Statistical analysis was performed using STATA V.15 software. Variables are expressed as a percentage (\%) for frequencies and mean $\pm \mathrm{SD}$ deviation for normally distributed continuous variables. Student's t-test was used to compare continuous variables between the two groups. Univariate analysis to compare categorical data was performed using the $\chi^{2}$ test. Multiple logistic regression analysis was performed to examine if the timing of diagnosis of GDM was independent risk factor for macrosomia, LGA, preterm delivery, C-section (caesarean section) and neonatal hypoglycaemia. $\mathrm{P}$ value $<0.05$ was considered significant.

\section{Patient and public involvement}

There is no patient and public involvement in this study.

\section{RESULTS}

There were 273 patients diagnosed with E-GDM and 528 patients with U-GDM. Table 1 shows the baseline characteristics of each group. E-GDM patients were significantly older (mean age was $33.5 \pm 5.4$ years vs $32.0 \pm 5.4$ years, $\mathrm{p}<0.001)$ and had higher BMI $(33.0 \pm 6.3$ vs $31.7 \pm 6.1$, $\mathrm{p}=0.0059)$ compared with U-GDM. There was no difference in ethnicity or in the prevalence of overweight and obesity between the two groups.

As shown in table 2, most of the patients in the two groups were screened using $75 \mathrm{~g}$ OGTT rather than 
Table 1 Baseline characteristics. Data are expressed as mean \pm SD or actual number of subjects and percentages

\begin{tabular}{|c|c|c|c|}
\hline & E-GDM (273) & U-GDM (528) & $P$ value \\
\hline Age (years) & $33.5 \pm 5.4$ & $32.0 \pm 5.4$ & $<0.001$ \\
\hline Prepregnancy weight* (kg) & $82.4 \pm 16.7$ & $79.7 \pm 16.4$ & 0.0308 \\
\hline Prepregnancy BMI categories & & & 0.069 \\
\hline Normal & $20(7.3 \%)$ & $60(11.7 \%)$ & \\
\hline Obese $(\geq 30)$ & $176(64.5 \%)$ & $293(57.3 \%)$ & \\
\hline Ethnicity & & & 0.524 \\
\hline Qatari & $112(41.0 \%)$ & $223(42.3 \%)$ & \\
\hline Non-Qatari-Arab & 89 (32.6\%) & 147 (27.8\%) & \\
\hline Asian & $64(23.4 \%)$ & $141(26.7 \%)$ & \\
\hline
\end{tabular}

* $2.2 \%$ of data are missing.

E-GDM, early gestational diabetes mellitus; U-GDM, usual gestational diabetes mellitus.

FBG alone. The mean gestational age at screening was $17.7 \pm 5.5$ weeks in the E-GDM group compared with $28.3 \pm 3.6$ weeks in the U-GDM group $(\mathrm{p}<0.001)$. The E-GDM group had higher FBG $(5.3 \pm 0.7 \mathrm{mmol} / \mathrm{L}$ vs $4.0 \pm 0.7 \mathrm{mmol} / \mathrm{L} ; \mathrm{p}<0.001)$, higher 1 hour post OGTT blood glucose $(10.6 \pm 1.7$ vs $10.3 \pm 1.6 \mathrm{mmol} / \mathrm{L}, \mathrm{p}=0.0198)$ but similar 2hour post OGTT blood glucose $(9.1 \pm 5.5$ vs $8.6 \pm 1.7 \mathrm{mmol} / \mathrm{L} ; \mathrm{p}=0.09$ ). Abnormal $\mathrm{FBG}$ was more prevalent in the E-GDM than in the U-GDM group ( $66.4 \%$ vs $45.6 \% ; \mathrm{p}<0.001)$. There was no difference in the frequency of abnormal 1 and 2 hours blood glucose post OGTT between the two groups. Compared with U-GDM, more patients in the E-GDM group required medications $(72.5 \%$ vs $46.0 \%$; $\mathrm{p}=0.001)$ and supplemental insulin metformin ( $38.4 \%$ vs $25.7 \%$; $\mathrm{p}=0.004)$.

Table 3 summarises the main maternal and fetal outcomes. The E-GDM group gained significantly less weight compared with U-GDM ( $\mathrm{wGWG}=0.021 \mathrm{~kg} /$ week vs $0.12 \mathrm{~kg} /$ week, respectively; $\mathrm{p}=0.0274$ ). There were no significant differences between the two groups in the rates of hypertensive disorders, polyhydramnios, recurrent vaginal infections, recurrent UTI, induction of labour, LGA, SGA, macrosomia, stillbirth, shoulder dystocia, neonatal hypoglycaemia and neonatal jaundice. E-GDM had higher incidence of preterm labour (25.5\% vs $14.4 \%, \mathrm{p}<0.001)$, C-section $(52.4 \%$ vs $42.8 \%$, $\mathrm{p}=0.010$ ), and NICU (neonatal intensive care) admissions $(17.6 \%$ vs $10.2 \%, \mathrm{p}<0.001)$ compared with U-GDM, respectively.

Table 4 shows multivariate logistic regression analysis for the identification of factors associated with macrosomia, LGA, C-section and neonatal hypoglycaemia. Prepregnancy maternal weight was an independent risk factor for these adverse outcomes. Age was associated with increased risk

Table 2 Glucose screening results and antidiabetic treatment. Data are expressed as mean \pm SD or actual number of subjects and percentages

\begin{tabular}{lllc}
\hline & E-GDM (273) & U-GDM (528) & P value \\
\hline FBG only & $36(14.8 \%)$ & $84(15.9 \%)$ & \\
OGTT & $207(85.2 \%)$ & $444(84.1 \%)$ & \\
Gestational age at time of OGTT (weeks) & $17.7 \pm 5.5$ & $28.3 \pm 3.6$ & $<0.001$ \\
FBG (mmol/L) & $5.3 \pm 0.7$ & $4.0 \pm 0.7$ & $<0.001$ \\
1 hour BG (mmol/L) & $10.6 \pm 1.7$ & $10.3 \pm 1.6$ & 0.0198 \\
2 hours (mmol/L) & $9.1 \pm 5.5$ & $8.6 \pm 1.7$ & 0.0909 \\
Diet only & $75(27.5 \%)$ & $283(53.6 \%)$ & $<0.001$ \\
Metformin only & $107 / 198(54.4 \%)$ & $156 / 245(63.7 \%)$ & 0.040 \\
Metformin plus insulin & $76 / 198(38.4 \%)$ & $63 / 245(25.7 \%)$ & 0.004 \\
\hline Insulin only & $15 / 198(7.6 \%)$ & $26 / 245(10.6 \%)$ & 0.228 \\
\hline
\end{tabular}

E-GDM, early gestational diabetes mellitus; FBG, fasting blood glucose; OGTT, oral glucose tolerance test; U-GDM, usual gestational diabetes mellitus. 
Table 3 Maternal and neonatal outcomes. Data are expressed as mean \pm SD or actual number of subjects and percentages

\begin{tabular}{lllc}
\hline & E-GDM (273) & U-GDM (528) & P value \\
\hline wGWG (kg/week) & $0.02 \pm 0.03$ & $0.12 \pm 0.03$ & 0.0274 \\
PIH & $16(5.86 \%)$ & $22(4.2 \%)$ & 0.285 \\
\hline Pre-eclampsia & $6(2.2 \%)$ & $20(3.8 \%)$ & 0.229 \\
\hline Polyhydramnios & $14(5.6 \%)$ & $32(6.5 \%)$ & 0.653 \\
\hline Recurrent UTI & $14(5.1 \%)$ & $18(3.4 \%)$ & 0.239 \\
\hline Recurrent vaginal infection & $3(1.1 \%)$ & $10(1.9 \%)$ & 0.339 \\
\hline GA at delivery (weeks) & $37.4 \pm 2.99$ & $38.4 \pm 2.09$ & $<0.001$ \\
\hline Induction of labour & $42(15.4 \%)$ & $96(18.2 \%)$ & 0.320 \\
\hline Preterm labour & $67(25.5 \%)$ & $76(14.4 \%)$ & $<0.001$ \\
\hline C-section & $143(52.4 \%)$ & $226(42.8 \%)$ & 0.010 \\
\hline Primary C-section & $59(21.6 \%)$ & $105(19.9 \%)$ & 0.327 \\
\hline Emergency C-section & $72(26.4 \%)$ & $109(20.6 \%)$ & 0.066 \\
\hline Neonatal weight (kg) & $3.086 \pm 0.76$ & $3.208 \pm 0.63$ & 0.0142 \\
LGA & $38(15.8 \%)$ & $73(15.5 \%)$ & 0.925 \\
\hline Macrosomia & $13(5.7 \%)$ & $44(9.3 \%)$ & 0.107 \\
\hline SGA & $32(13.6 \%)$ & $57(12.5 \%)$ & 0.686 \\
\hline Stillbirth & $1(0.7 \%)$ & $4(0.8 \%)$ & 0.458 \\
NICU admission & $48(17.6 \%)$ & $54(10.2 \%)$ & 0.003 \\
\hline Shoulder dystocia & $1(0.7 \%)$ & $1(0.2 \%)$ & 0.634 \\
Respiratory distress & $31(7.0 \%)$ & $30(8.4 \%)$ & 0.013 \\
Neonatal hypoglycaemia & $15(5.5 \%)$ & $47(8.9 \%)$ & 0.087 \\
Neonatal jaundice & $43(15.8 \%)$ & $73(13.8 \%)$ & 0.463 \\
\hline
\end{tabular}

GA, gestational age; LGA, large for gestational age; NICU, neonatal intensive care; PIH , pregnancy -induced hypertension ; SGA, small for gestational age; wGWG, weekly gestational weight gain. UTI, urinary tract infection.

for macrosomia and C-section. Gestational weight gain was associated with increase odds for both macrosomia and LGA. The time of diagnosis of GDM was associated with higher risk of macrosomia and neonatal hypoglycaemia, that is, the later the diagnosis of GDM, the higher the risks of macrosomia and neonatal hypoglycaemia.

\section{DISCUSSION}

This study is the first to report outcomes of early detected GDM versus usual GDM in the Middle East and North
Africa region. This study has shown that patients with E-GDM are older, more likely to be obese, more likely to need treatment and more likely to need supplemental insulin therapy compared with patients with U-GDM. There was no difference in most of the maternal and neonatal outcomes between the two groups apart from higher rates of preterm deliveries, C-section and NICU admission in the E-GDM group compared with U-GDM group. Multivariate analysis showed that the timing of diagnosis of GDM is an independent risk factor for

Table 4 Multivariate logistic regression analysis

\begin{tabular}{|c|c|c|c|c|}
\hline & Macrosomia & LGA & C-section & Neonatal hypoglycaemia \\
\hline & AOR $(95 \% \mathrm{Cl})$ & AOR $(95 \% \mathrm{Cl})$ & AOR $(95 \% \mathrm{Cl})$ & AOR $(95 \% \mathrm{Cl})$ \\
\hline Age & $1.05(1.00 \text { to } 1.12)^{\star}$ & 1.02 (0.98 to 1.07$)$ & $1.07(1.04 \text { to } 1.11)^{*}$ & 0.98 (0.9 to 1.04$)$ \\
\hline GWG & $1.07(1.03 \text { to } 1.12)^{\star}$ & $1.04(1.01 \text { to } 1.09)^{*}$ & 1.02 (0.99 to 1.04) & 0.98 (0.93 to 1.02$)$ \\
\hline GA-OGTT & $1.06(1.00 \text { to } 1.11)^{\star}$ & 1.00 (0.97 to 1.04$)$ & $0.98(0.96$ to 1.01$)$ & $1.05(1.00 \text { to } 1.11)^{\star}$ \\
\hline
\end{tabular}

${ }^{*} \mathrm{P}<0.05$

AOR, adjusted odds' ratio; GA-OGTT, gestational age at the time of OGTT screening; GWG, gestational weight gain; LGA, large for gestational age. 
macrosomia and neonatal hypoglycaemia; after correction for known risk factors.

The literature shows controversy on the outcomes of early gestational diabetes. The earlier studies used different glucose cut-off points to diagnose E-GDM; used different gestational ages to define E-GDM; did not correct for confounders and the adverse pregnancy outcomes were not consistently defined. Sweeting et al examined a large database of 4873 women with GDM (FBG $\geq 5.5 \mathrm{mmol} / \mathrm{L}$ and 2 hours $\geq 8.0 \mathrm{mmol} / \mathrm{L}$ ) over a period of 20 years between 1991 and 2011 and categorised them into three groups based on the time of diagnosis; $<12$ weeks of gestation, 12-23 weeks of gestation and $\geq 24$ weeks of gestation. ${ }^{7}$ The study showed that hypertensive disorders in pregnancy, preterm delivery, C-section, macrosomia and LGA were more prevalent in women with E-GDM than in U-GDM. The study did not correct for changes in important confounders over this period of time; namely baseline maternal BMI and age of the mother at conception. Yet, the study undertook regression analysis for LGA and macrosomia, demonstrating that prepregnancy BMI, GWG, fasting OGTT value and C-section were independent risk factors for macrosomia, while prepregnancy BMI, GWG, C-section and hypertensive disorders of pregnancy were independent risk factors for LGA. A smaller study of 120 women with GDM (FBG $\geq 5.3 \mathrm{mmol} / \mathrm{L}$ and 2 hours $\geq 8.6 \mathrm{mmol} / \mathrm{L}$ ) showed that E-GDM was associated with an increased risk of hypertensive disorders in pregnancy, NICU admissions, neonatal hypoglycaemia and perinatal death compared with U-GDM but not with other pregnancy outcomes. ${ }^{11}$ Alunni et al compared E-GDM (FBG $\geq 5.1 \&$ / or HBA1C $\geq 5.7 \%$ ) versus U-GDM (FBG $\geq 5.3 \mathrm{mmol} / \mathrm{L}$ and 2 hours $\geq 8.6 \mathrm{mmol} / \mathrm{L}$ ) and found no difference in outcomes between the two groups apart from a greater need for pharmacotherapy in patients with E-GDM. ${ }^{12}$ A recent meta-analysis of cohort studies showed E-GDM patients had higher risk of neonatal mortality, neonatal hypoglycaemia and insulin treatment compared with U-GDM. ${ }^{9}$

GDM is characterised by impaired pancreatic $\beta$-cell function that is insufficient to overcome the insulin resistance that occur at the second half of pregnancy, which is multifactorial and is largely influenced by placental hormones. ${ }^{13}$ The FBG was significantly higher in the E-GDM compared with U-GDM suggesting that insulin resistance existed in the E-GDM before pregnancy. Harreiter et al performed OGTT at a median of 15 weeks' gestation in women with BMI $>29 \mathrm{~kg} / \mathrm{m}^{2}$ and showed that women with early GDM had a significantly high level of insulin resistance and $\beta$-cell dysfunctionafter correcting for age, pregestational BMI, gestational week, and fetal sex-compared with normoglycaemic women. ${ }^{14}$ Bozkurt et al showed that women with early GDM had a higher degree of insulin resistance but a similar degree of impaired $\beta$-cell function compared with women with late-onset GDM. ${ }^{15}$ Taken together, FBG could be the best screening test to detect early onset GDM. Prospective studies are needed to examine this further.
Our study is the first to describe a positive influence of the timing of diagnosis of GDM on pregnancy outcomes. In our study, the later the diagnosis of GDM, the higher the risks of macrosomia and neonatal hypoglycaemia even after correction for age, initial weight, gestational weight gain and the use of metformin. This finding was surprising as both macrosomia and neonatal hypoglycaemia are markers of fetal hyperinsulinaemia resulting mostly from maternal hyperglycaemia. It is more likely that the early-onset of hyperglycaemia leads to higher risk of fetal hyperinsulinaemia rather than vice-versa. One possibility is that early intervention in the E-GDM by modifying diet and introducing metformin reduces the fetal glucose steal that develops early in gestation and hence reduces the fetal hyperinsulinaemia. ${ }^{16}$ The earlier intervention with diet and metformin could also have reduced the gestational weight gain in early pregnancy, which is an independent risk factor for macrosomia. ${ }^{17}$ Indeed, metformin use in our study was associated with $56 \%$ reduction in neonatal hypoglycaemia which was previously described in other studies. ${ }^{18}{ }^{19}$ However, the mean gestational age at which OGTT was performed was 17 weeks, hence early intervention cannot solely explain the results. In addition, $90 \%$ of our GDM population were overweight and obese at conception and early blood glucose levels even within the higher end of the normal spectrum could have triggered fetal hyperinsulinaemia earlier than expected. This was shown in a study which classified early-pregnancy FBG results from 6129 patients into seven groups similar to the HAPO trial. ${ }^{50}$ The frequency of LGA and/or macrosomia ranged between $10 \%$ and $12 \%$ for the FBG levels between 4.5 and $5.1 \mathrm{mmol} / \mathrm{L}$ raising questions about the proper definition of early gestational diabetes. Finally, some of the patients in the U-GDM group might have not been screened early in pregnancy and hence the higher rates of macrosomia and neonatal hypoglycaemia.

The main limitations of our study are the retrospective design; the duration between diagnosis and intervention was not available; the rate of gestational weight gain in each trimester was not available and the glycaemic control data were not captured. Furthermore, the screening for GDM in the first trimester might have not been performed in some of the patients in the U-GDM group. On the other hand, a major strength is the large number of the study population and the low level of missing data. In addition, this is a single-centre study and both groups were treated by the same multidisciplinary team using standard protocols, this eliminating treatment bias.

\section{CONCLUSION}

In conclusion, our data support the concept of early screening for gestational diabetes in high-risk patient as early diagnosis and intervention is associated with a better outcome. Preconception weight, gestational weight gain and the timing of diagnosis of GDM are all independent 
risk factors for macrosomia. While we believe that early screening can reduce the risk of macrosomia, it is still not clear what is the optimum glucose cut-off points that are required to diagnose GDM in early pregnancy. Further prospective studies are needed to explore the best timing of screening, and the glucose cut-off points to diagnose e-GDM.

Contributors MB designed the study, the data collection sheet, performed the statistical analysis and interpretation of data, and drafted the manuscript. KB, EN, FE and NA collected the data and reviewed the manuscript. Both Professors JCK and A-BA-S participated in the study design, data interpretation and reviewed the manuscript.

Funding The authors have not declared a specific grant for this research from any funding agency in the public, commercial or not-for-profit sectors.

Competing interests None declared.

Patient consent for publication Not required.

Ethics approval The study was approved by the Internal Review Board (IRB), Hamad Medical Corporation.

Provenance and peer review Not commissioned; externally peer reviewed.

Data sharing statement All data generated or analysed during this study are included in this published article. We do not have any other supplementary data.

Open access This is an open access article distributed in accordance with the Creative Commons Attribution Non Commercial (CC BY-NC 4.0) license, which permits others to distribute, remix, adapt, build upon this work non-commercially, and license their derivative works on different terms, provided the original work is properly cited, appropriate credit is given, any changes made indicated, and the use is non-commercial. See: http://creativecommons.org/licenses/by-nc/4.0/.

\section{REFERENCES}

1. World Health Organization. Diagnostic criteria and classification of hyperglycaemia first detected in pregnancy. http://apps.who.int/iris/ bitstream/10665/85975/1/WHO_NMH_MND_13.2_eng.pdf (Accessed 30 Jan 2018).

2. ADA. 2. Classification and diagnosis of diabetes. Diabetes Care 2014;38:S8-S16.

3. Blumer I, Hadar E, Hadden DR, et al. Diabetes and pregnancy: an endocrine society clinical practice guideline. J Clin Endocrinol Metab 2013;98:4227-49.
4. NICE guideline. Diabetes in pregnancy: management from preconception to the postnatal period. 2015 https://www.nice.org.uk/ guidance/ng3

5. Hyperglycemia and Adverse Pregnancy Outcomes (HAPO). 2008

6. American Diabetes Association. 2. Classification and diagnosis of diabetes: Standards of Medical Care in Diabetes-2018. Diabetes Care 2018;41(Suppl 1):S13-27.

7. Sweeting AN, Ross GP, Hyett J, et al. Gestational diabetes mellitus in early pregnancy: evidence for poor pregnancy outcomes despite treatment. Diabetes Care 2016;39:75-81.

8. Boriboonhirunsarn D, Kasempipatchai V. Incidence of large for gestational age infants when gestational diabetes mellitus is diagnosed early and late in pregnancy. J Obstet Gynaecol Res 2016;42:273-8.

9. Immanuel J, Simmons D. Screening and treatment for early-onset gestational diabetes mellitus: a systematic review and meta-analysis. Curr Diab Rep 2017;17:115.

10. Al-Thani AA, Bakri AH. Qatar Stepwise Report 2012 [Chronic Disease Risk Factor Surveillance]. 2012:1-124.

11. Easmin S, Chowdhury TA, Islam MR, et al. Obstetric outcome in early and late onset gestational diabetes mellitus. Mymensingh Med J 2015;24:450-6

12. Alunni ML, Roeder HA, Moore TR, et al. First trimester gestational diabetes screening - change in incidence and pharmacotherapy need. Diabetes Res Clin Pract 2015;109:135-40.

13. Buchanan TA, Xiang A, Kjos SL, et al. What Is Gestational Diabetes? Diabetes Care 2007;30:S105-11.

14. Harreiter J, Simmons D, Desoye G, et al. IADPSG and WHO 2013 gestational diabetes mellitus criteria identify obese women with marked insulin resistance in early pregnancy. Diabetes Care 2016;39:e90-2.

15. Bozkurt L, Göbl CS, Pfligl L, et al. Pathophysiological characteristics and effects of obesity in women with early and late manifestation of gestational diabetes diagnosed by the international association of diabetes and pregnancy study groups criteria. J Clin Endocrinol Metab 2015;100:1113-20.

16. Desoye G, Nolan CJ. The fetal glucose steal: an underappreciated phenomenon in diabetic pregnancy. Diabetologia 2016;59:1089-94.

17. Clausen T, Burski TK, Øyen N, et al. Maternal anthropometric and metabolic factors in the first half of pregnancy and risk of neonatal macrosomia in term pregnancies. A prospective study. Eur $J$ Endocrinol 2005;153:887-94.

18. Kitwitee P, Limwattananon S, Limwattananon C, et al. Metformin for the treatment of gestational diabetes: an updated meta-analysis. Diabetes Res Clin Pract 2015;109:521-32.

19. Butalia S, Gutierrez L, Lodha A, et al. Short- and long-term outcomes of metformin compared with insulin alone in pregnancy: a systematic review and meta-analysis. Diabet Med 2017;34:27-36.

20. Riskin-Mashiah S, Younes G, Damti A, et al. First-trimester fasting hyperglycemia and adverse pregnancy outcomes. Diabetes Care 2009;32:1639-43. 